SISOMOS

AMERICANOS

Revista de Estudios

Transfronterizos
Volumen XXI, número I,

enero-junio de 2021

Recibido: 29 de abril de 2020

Aprobado: 20 de enero de 2021

\title{
ZICOSUR, paradiplomacia y recursos naturales: el litio y la vinculación con China*
}

\section{ZICOSUR, paradiplomacy and natural resources: Lithium and the \\ relationship with China}

\author{
Stella Juste ${ }^{* *}$
}

Universidad Nacional de Jujuy, Argentina.

\begin{abstract}
Resumen
El presente artículo propone el análisis de los bienes naturales de la Zona de Integración del Centro Oeste de América del Sur (ZICOSUR), específicamente del litio, en relación con su potencial regional en el proceso de vinculación con China. Para tal fin se aborda el estudio de los recursos naturales de la región, que incluye las subregiones del Gran Chaco y del "triángulo del litio". ZICOSUR se caracteriza por ser un proceso de integración subestatal integrado, mayormente, por unidades subestatales periféricas que persiguen una inserción internacional como región, ampliando sus posibilidades de desarrollo. En este sentido, el trabajo propone el examen de la complementariedad de intereses con el gigante asiático, que puedan conducir a relaciones de cooperación. El
\end{abstract}

* El artículo es parte del proyecto posdoctoral "Grandes potencias y gestión transnacional de las provincias argentinas. Oportunidades y dess de la complementariedad con China en el desarrollo energético local y sus consecuencias sobre las comunidades locales (2014-2018)".

Doctora en Relaciones Internacionales, Universidad Nacional de Rosario (Argentina). Profesora, Universidad Nacional de Jujuy, Argentina. Becaria posdoctoral del Consejo Nacional de Investigaciones Científicas y Técnicas (CONICET). Miembro de la Red de Expertos en Paradiplomacia e Internacionalización Territorial (REPIT). Dirección postal: Doctor Baldi 1660, San Salvador de Jujuy, Argentina. Correo electrónico: msjuste@cisor.unju.edu.ar ORCID: https://orcid.org/0000-0002-14756074

Cómo citar este artículo: Juste, Stella (2021) ZICOSUR, paradiplomacia y recursos naturales: el litio y la vinculación con China. Si Somos Americanos. Revista de Estudios Transfronterizos, 21(1), 7-31. doi: $10.4067 /$ S0719-09482021000100007 
marco teórico propuesto parte de los estudios de paradiplomacia para entender el accionar de las unidades subestatales, y de los estudios de integración regional para comprender el fenómeno a nivel subestatal en relación con la gestión de los recursos naturales. La propuesta metodológica es principalmente cualitativa con estudio de caso, y también basada en el análisis de documentos oficiales y fuentes bibliográficas.

Palabras clave: integración regional subnacional, China, litio.

\begin{abstract}
This article analyses the natural assets of the Area of Integration of Central West South America (Spanish acronym, ZICOSUR), specifically lithium, with respect to its regional potential in the process of linking up with China. The authors examine the natural resources of the region, which includes the sub-regions of Gran Chaco and the "lithium triangle". ZICOSUR is a sub-state integration process that mainly involves peripheral sub-state units seeking regional international insertion in order to expand their development possibilities. In this respect, the work examines the complementarity of interests with the Asian giant, which may lead to cooperative relations. The proposed theoretical framework begins with paradiplomacy studies to understand the actions of sub-state units, and from regional integration studies to understand the phenomenon at the sub-state level in relation to the management of natural resources. The methodological proposal is mainly qualitative, employing a case study and the analysis official documents and bibliographic sources.
\end{abstract}

Keywords: subnational regional integration, China, lithium.

\title{
Introducción
}

Durante las últimas décadas, y ante un mundo globalizado y competitivo, con actores diversos y dinámicas complejas, han proliferado iniciativas de integración en distintos puntos del mundo. A través de la cooperación y la puesta en común de objetivos y problemáticas han surgido iniciativas que buscan optimizar los escenarios y las capacidades internas, de cara a mejorar las condiciones de inserción internacional. Entre estas se destacan la Unión Europea en el Viejo Continente, el Mercado Común del Sur (MERCOSUR) en Sudamérica, y la Asociación de Naciones del Sudeste Asiático (ASEAN) en el Sudeste asiático. A nivel subestatal, la integración ha interpelado a las unidades subestatales -departamentos, provincias, estados federados, entre otros-, dando lugar a iniciativas de integración de carácter subestatal en zonas transfronterizas (Venegas San Martín, 2019). La proximidad territorial y la confluencia de intereses y problemáticas observadas en los territorios transfronterizos fueron factores que promovieron el avance de la integración. Las unidades subestatales colindantes, aun cuando pertenecen a distintos países, buscan alternativas a problemas compartidos, la 
disminución de asimetrías y la competitividad frente a terceros. Autores como Valle Sosa (2012) sostienen que las iniciativas de integración subestatal "se encuentran determinadas por los vínculos de proximidad geográfica, cultural, histórica y económica, como así también similares problemáticabicación periférica, lejanía con los centros de comercialización, menos desarrollo relativo, etcétera)" (2012, p. 78). Esta situación las motiva a construir escenarios de cooperación para el fortalecimiento regionaovechando factores como la posición geográfica, las condiciones ambientales, las inversiones extranjeras y la voluntad política.

En este marco contextual, la Zona de Integración del Centro Oeste de América del Sur (ZICOSUR) es una iniciativa de unidades subestatales que nació en 1997, impulsada de forma conjunta por los gobiernos locales y el sector privado. Su objetivo inicial fue alcanzar el desarrollo sustentable y la inserción de la subregión en el contexto internacional, promoviendo el comercio exterior. Actualmente, está integrada por unidades subestatales de siete países: Argentina, Bolivia, Brasil, Chile, Paraguay, Perú y Uruguay.

Las unidades subestatales que forman la ZICOSUR pertenecen -mayormente- a países que integran el MERCOSUR, es decir, a Argentina, Brasil, Paraguay, Uruguay y Bolivia. ${ }^{1}$ Por lo tanto, es observable que la voluntad de integrarse es una visión multinivel compartida en Sudamérica, tanto por los Estados como así también por las unidades subestatales que lo componen. Pero, a diferencia del MERCOSUR, la ZICOSUR nace por iniciativa y decisión propias de unidades subestatales argentinas, bolivianas, brasileñas, chilenas y paraguayas a las que tiempo más ta se sumarían otras. No hay intervención de los Estados y de los gobiernos nacionales en este proceso.

En números, la ZICOSUR se compone de un total de 75 unidades subestatales pertenecientes a los países antes mencionados. Entre todas concentran una población cercana a los 77 millones de habitantes en una superficie de cinco millones de kilómetros cuadrados. Las provincias, departamentos, regiones y estados que la integran son, en su mayoría, economías de menor desarrollo relativo dentro de sus Estados, detectándose carencias en infraestructura y lejanía de los centros de distribución y consumo, así como bajos niveles de industrialización (ver Mapa $\mathrm{N}^{\circ} 1$ ).

\footnotetext{
${ }^{1}$ Cabe destacar que Bolivia se encuentra en proceso de adhesión al MERCOSUR. Si bien el protocolo de adhesión fue firmado por los Estados Partes en 2015, está en vías de incorporación por los Congresos de estas naciones.
} 


\section{Mapa $\mathbf{N}^{\circ}$ 1: La subregión de la ZICOSUR}

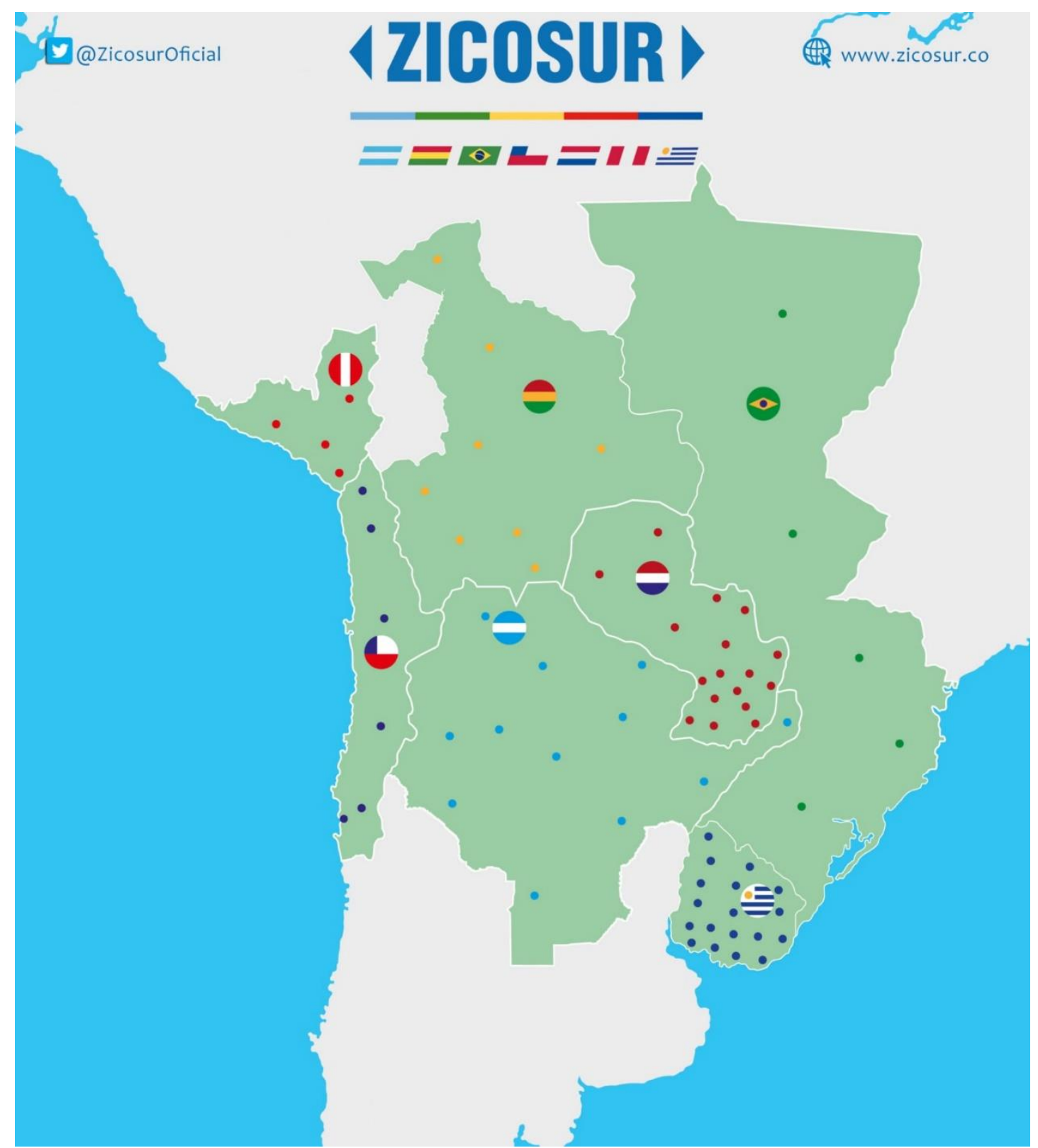

Fuente: ZICOSUR (23 de diciembre de 2020).

El inicio de la Zicosur estuvo marcado por el impulso y la presión de unidades subestatales periféricas de países periféricos en el sistema internacional. Es decir, fue constituida inicialmente por unidades subestatales de doble periferia (Juste, 2017a). Pero a medida que la integración fue avanzando, incorporó a otras unidades de mayor centralidad como Córdoba y Santa Fe en Argentina, Santa Cruz de la Sierra en Bolivia, Rio Grande do Sul y Santa Caterina en Brasil, y Colonia y Montevideo en Uruguay.

La inserción periférica de algunos Estados en el sistema internacional, se reproduce hacia el interior de las fronteras estatales, favoreciendo la aparición de unidades subestatales de centro y unidades subestatales periféricas. A partir 
de allí, pueden identificarse unidades subestatales de doble periferia, es decir, periféricas respecto del Estado que forman parte, y respecto del Sistema Internacional.(Juste,2017a,p171) ${ }^{2}$

La doble periferia es una situación política de doble dependencia en que se encuentra una unidad subestatal respecto del centro propulsor internacional y al interior del Estado periférico de pertenencia. Esta condición de doble periferia se ve reflejada en una serie de factores tales como el aislamiento geográfico, la ausencia de liderazgos, la inestabilidad de las instituciones, las limitaciones en la cohesión y movilidad social, la incapacidad para pensar un modelo de desarrollo propio, y la debilidad en una articulación productiva del territorio que incluya cadenas de valor transfronterizas. (Juste y Oddone, 2020, p. 73)

$\mathrm{Su}$ origen se relaciona con la idea de fortalecer la franja territorial del centro-oeste sudamericano, con el fin de potenciar la conexión bioceánica de esa región y, sobre todo, los puertos del Pacífico que conectan con los mercados asiáticos. Prueba de ello es que los primeros encuentros oficiales tuvieron la presencia de autoridades diplomáticas y del sector privado de países asiáticos. Esta idea plantea numerosos interrogantes sobre la articulación de la ZICOSUR y el MERCOSUR respecto de la integración, en escenarios con alta complejidad. Esto es porque la ZICOSUR es un proceso de integración subregional ${ }^{3}$ subestatal intra-MERCOSUR, lo que permite pensar en tensiones relacionadas con la desarticulación o falta de representación de los objetivos de desarrollo subestatal que no han sido canalizados en el proceso de integración interestatal, dando lugar al surgimiento de una iniciativa subestataln este desafío doble que se propuso ZICOSUR -profundizar la integración "hacia adentro" y vincularse con Asia Pacífico-, las unidades subestatales fueron conscientes de que, para alcanzar el segundo objetivo, debían concretar antes el primero. Entonces, trabajar la integración de la subregión se convirtió en el principal objetivo.

Los territorios de esta subregión se caracterizan por su riqueza natural, pues cuentan con importantes reservorios energéticos, especialmente de gas, petróleo y litio. Entre ellos, la región del Gran Chaco aparece como una zona de "reserva energética", a la que se suma el triángulo del litio, ubicado en el norte argentino, norte chileno y sur boliviano. Además, de ser economías primarias, distantes de las capitales (mayores puntos de consumo) y de los puertos y centros de distribución, presentan debilidades en infraestructura de conectividad (carreteras, líneas ferroviarias, vuelos, entre otros), hecho que impide un flujo adecuado de bienes, servicios y personas.

Los lazos históricos que unen a estos territorios son múltiples, y combinan la riqueza de la cultura andina en el oeste con el patrimonio de las yungas y selvas hacia el este. Esta confluencia de factores hace de la subregión un área única en diversidad de riquezas

\footnotetext{
${ }^{2}$ Para una mayor profundización sobre la conceptualización de "doble periferia", se recomienda consultar Juste (2017a).

${ }^{3}$ Se consideran "subregiones" a las iniciativas de integración subestatal como la ZICOSUR, mientras que las iniciativas de integración de carácter inter-estatal, como el MERCOSUR, la Unión Europea, la ASEAN, etc., son denominadas "regiones".
} 
naturales y culturales. Aun con esas diferencias, comparten un núcleo de problemáticas $\mathrm{y}$ prioridades que han contribuido a diseñar acciones coordinadas para mejorar sus posibilidades de desarrollo.

Geográficamente, cuentan con un elemento que favorece su inserción internacional: su carácter de subregión bioceánica. Los puertos del norte chileno brindan salida hacia el océano Pacífico, y los puertos del sur brasileño hacia el océano Atlántico. Este factor es clave para la vinculación con los puertos asiáticos, sin dejar de lado las relaciones comerciales tradicionales de Sudamérica con Europa.

Del otro lado del mundo se encuentran los países emergentes asiáticos. Particularmente China, en ascenso económico y político, precisa asegurarse los recursos naturales necesarios que garanticen la continuidad de su crecimiento. El país asiático propició inversiones en proyectos relacionados con recursos energéticos, mineros y agrícolas en distintos puntos de la geografía latinoamericana (Juste, 2017b). En este sentido, su importancia para el sector energético de la región se observa en tres dimensiones: inversiones directas, préstamos bancarios e intercambio comercial. Además, China es hoy el primer consumidor de litio a nivel mundial, principalmente para la elaboración de baterías (López, Obaya, Pascuini y Ramos, 2019).

Con este marco precedente, ¿son complementarios los intereses de la ZICOSUR con los intereses chinos en la región? ¿En qué condiciones la explotación de las riquezas naturales de la ZICOSUR constituye una posibilidad de desarrollo a largo plazo?

La hipótesis del presente artículo plantea que el desarrollo de una matriz productiva extractiva alrededor de los recursos naturales de la ZICOSUR, especialmente el litio, posibilitará una vinculación complementaria a los intereses de China. Sin embargo, si la ZICOSUR avanza sobre la conformación de una cadena de valor regional alrededor del litio, diversificando su producción primaria, debilitará la complementariedad y el vínculo con China a largo plazo.

El Manual para el fortalecimiento de cadenas de valor de la CEPAL (Padilla Pérez y Oddone, 2016) sostiene que una cadena de valor "comprende la amplia variedad de actividades para que un producto o servicio transite a través de diferentes etapas, desde su concepción hasta su entrega a los consumidores y la disposición final después de su uso" (2016, p. 17). En este proceso, cada una de esas etapas (diseño, producción, tránsito de la mercancía, consumo, etc.) recibe el nombre de eslabón.

Las cadenas regionales de valor constituyen un proceso de encadenamiento productivo transfronterizo en el que participan dos o más países con afinidad productiva, cercanía territorial y complementariedad comercial, con el objetivo de alcanzar una mayor articulación a partir de la integración de los diferentes eslabones de uno y otro lado de la(s) frontera(s). De esta manera, el valor "es agregado" por diversos países cercanos con características económicas comunes o complementarias. (CENPROMYPE, CEPAL y SIECA, 2018, p. 16).

Por otro lado, y en referencia al campo académico de las relaciones internacionales, el accionar internacional de las unidades subestatales despertó interés en la década de 
1980. En ese momento, la concepción interestatal del sistema internacional y la idea del Estado como único actor empezaron a cuestionarse ante el avance de otros actores como la sociedad civil organizada, el sector privado y los gobiernos locales, entre otros. Esta nueva situación fue explicada por el enfoque de la "interdependencia compleja", que surge como crítica a la corriente realista y se sustenta en el contexto global de transformación que propicia la aparición de actores de distinta naturaleza (Keohane y Nye, 1989).

Paralelamente y en sintonía con Keohane y Nye, Manning (1977) caracterizaba la realidad internacional como interméstica (intermestic), al plantear que la agenda internacional afectaba directamente a la realidad política y económica local. De acuerdo con Manning, "la naturaleza de los asuntos internacionales contemporáneos está marcada por ser simultánea, profunda e inseparablemente internacional cuanto doméstica" (1977, p. 309). También Gourevitch (1996) sostuvo que el sistema internacional "no es sólo una consecuencia de la política y estructuras domésticas, sino una causa de las mismas" (1996, pp. 23-24), siendo ese el motivo principal por el cual la política internacional y la doméstica están íntimamente relacionadas y no pueden ser analizadas por separado.

Esta apertura teórica dio pie al surgimiento de una nueva comunidad epistémica conocida como los "estudios de paradiplomacia". Específicamente, la expresión "paradiplomacia" fue propuesta por Duchacek (1990), que la definió como "las entradas directas e indirectas de los gobiernos no centrales al campo de las relaciones internacionales" (1990, p. 15), poniendo énfasis en el prefijo "para-", entendiendo que esta capacidad de los gobiernos locales era "paralela", es decir, "subsidiaria o accesoria" respecto de la política exterior de los Estados. Más tarde, Cornago Prieto (cit. en Maira, 2010), uno de sus principales impulsores, conceptualizó la paradiplomacia como:

La implicación de los gobiernos no centrales en las relaciones internacionales, a través del establecimiento de contactos formales e informales, permanentes o ad hoc con entidades extranjeras, públicas y privadas, con el propósito de promover asuntos de carácter socioeconómicos, políticos o culturales, así como cualquier otra dimensión externa de sus competencias constitucionales. (2010, pp. 27-28).

Más recientemente, Calvento (2014) profundizó en la temática a través del concepto de "gestión internacional", en el sentido de una política pública generada por actores gubernamentales subestatales a partir de una sinergia social, económica y política. Con tales premisas, define la gestión internacional como "una herramienta que impulsa la inserción internacional buscando aprovechar las oportunidades del contexto exterior y presentándose como un instrumento significativo para el desarrollo local" (2014, p. 304). Entonces, una de las modalidades que emplea el accionar internacional de las 
unidades subestatales es vincularse a través de iniciativas de integración con sus pares transfronterizos, objeto de este análisis. ${ }^{4}$

En este aspecto, la dimensión transfronteriza es entendida según Perkmann (2003) como "una colaboración entre autoridades subnacionales más allá de los límites fronterizos nacionales" (p. 157). Asimismo, Rhi-Sausi y Oddone (2009) plantean que la paradiplomacia transfronteriza "permite la participación y actuación conjunta y en forma de red de los actores públicos y privados del territorio a ambos lados de la frontera" (p. 37).

Sin embargo, cuando se observan los estudios de integración regional -sea en sentido económico, político o amplio-, la referencia suelen ser los procesos del tipo supranacional, es decir, la integración entre Estados (Venegas San Martín, 2019). Esto puede deberse a su importancia política, tamaño geográfico, volúmenes comerciales y hasta motivos históricos; entre ellos destacamos la Unión Europea, el MERCOSUR, la ASEAN, entre otros. Pero también es cierto que existen otras tipologías de integración en relación con los actores que la propulsan. Fenómenos como la globalización y la descentralización han promovido la integración de unidades subestatales a través de diferentes iniciativas.

La internacionalización de las unidades subestatales y su participación en procesos de integración merece múltiples reflexiones. Por un lado, pone en evidencia que los procesos de integración interestatales, en sus diferentes casos, no han conseguido generar beneficios importantes en las complejas y heterogéneas realidades estatales y subestatales. Por otro lado, expone la complejización del sistema internacional y la existencia de una multiplicidad de actores que despliegan su accionar en función de sus propios intereses y motivaciones.

\section{EI triángulo del litio, el reservorio energético de la ZICOSUR}

En el extremo occidental de la ZICOSUR encontramos otra subregión, conocida como el "triángulo del litio". Esta zona está conformada por el sur boliviano, el norte argentino y el norte chileno (Quinteros, 2020). Según datos del Servicio Geológico de Estados Unidos (USGS), alrededor del 67\% de las reservas probadas de litio y, alrededor de la mitad de la oferta global, se encuentran en esta región (López et al., 2019) (ver Cuadro $\mathrm{N}^{\circ} 1$ ).

\footnotetext{
4 Álvarez (2017) planteó que la paradiplomacia puede ser abordada estudiando sus causas, sus motivaciones y objetivos, la forma en que se lleva a cabo, o el canal que utiliza.
} 
Cuadro $\mathrm{N}^{\circ}$ 1: Distribución de los proyectos de litio en la ZICOSUR

\begin{tabular}{llll}
\hline PAÍS & UNIDAD & PROYECTO EMPRESA ORIGEN
\end{tabular}

\begin{tabular}{|c|c|c|c|c|}
\hline & & Cauchari- & $\begin{array}{l}\text { Lithium } \\
\text { Americas }\end{array}$ & Canadá \\
\hline & & & $\begin{array}{l}\text { Ganfeng } \\
\text { Lithium }\end{array}$ & China \\
\hline & & & JEMSE & $\begin{array}{l}\text { Argentina } \\
\text { (Jujuy) }\end{array}$ \\
\hline & & & Orocobre & Australia \\
\hline & JUJUY & Olaroz & $\begin{array}{l}\text { Toyota } \\
\text { Tsusho }\end{array}$ & Japón \\
\hline & & & JEMSE & $\begin{array}{l}\text { Argentina } \\
\text { (Jujuy) }\end{array}$ \\
\hline & & Pozuelos & $\begin{array}{l}\text { LSC } \\
\text { Lithium }\end{array}$ & Canadá \\
\hline & & & Lithea & \\
\hline & SALTA & Rincón & $\begin{array}{l}\text { Argos y } \\
\text { Minerals }\end{array}$ & Australia \\
\hline & & $\begin{array}{l}\text { Pastos } \\
\text { Grandes }\end{array}$ & Millenial & Canadá \\
\hline & CATAMARCA & Fénix & Livent & $\begin{array}{l}\text { Estados } \\
\text { Unidos }\end{array}$ \\
\hline & & Atacama & Arbemarle & $\begin{array}{l}\text { Estados } \\
\text { Unidos }\end{array}$ \\
\hline & ANTOFAGASTA & & $\begin{array}{l}\text { SQM - } \\
\text { Tianqi } \\
\text { Lithium }\end{array}$ & Chile/China \\
\hline & & Salar Blanco & Codelco & Chile \\
\hline Chile & & & $\begin{array}{l}\text { Lithium } \\
\text { Power }\end{array}$ & Australia \\
\hline & $\Delta \mathrm{T} A C \Delta \mathrm{MA}$ & $M 0$ & MSB & Chile \\
\hline & & & $\begin{array}{l}\text { Bearing } \\
\text { Lithium }\end{array}$ & Canadá \\
\hline Bolivia & POTOSÍ & Uyuni & YLB & Bolivia \\
\hline
\end{tabular}

Fuente: elaboración propia sobre la base de datos propios y de Quinteros (2020). 
En términos absolutos, el salar de Atacama (Chile) es el segundo depósito de litio del mundo (superado por el Salar de Uyuni en Bolivia), pero presenta una notable ventaja económica: es el de mayor concentración y calidad de litio y dispone de las mejores condiciones climáticas para la evaporación, lo que disminuye los costos de procesamiento (Juste, 2017b). Lo interesante de este reservorio no solo es la participación china en la producción del carbonato de litio, sino que, además, la producción de los proyectos de Jujuy, Salta y Potosí tienen como destino final China, aunque se produzcan bajo firmas de otro origen.

La relevancia del litio radica en su capacidad para almacenar energía necesaria para el funcionamiento de múltiples dispositivos electrónicos, medios de transporte, autopartes, entre otros. Es el mineral estrella de la actualidad y, siguiendo a Fornillo (2019), "se lo llamó oro blanco, petróleo del siglo XXI" (p. 16). Las reservas andinas han sido catalogadas como la "“Arabia Saudita del litio' dando la pauta de una serie de imaginarios que es preciso delimitar, a veces deconstruir" (2019, p. 16). No obstante, los tres países y las unidades subestatales donde los proyectos de litio se sitúan, persiguen estrategias diferentes para su explotación y aprovechamiento, producto de concepciones diferenciadas sobre sus recursos naturales.

En Chile, las empresas explotan el litio sobre la base de un acuerdo con el Estado nacional, siendo este país el principal productor en el mundo. En 2015, la administración de Michelle Bachelet conformó la Comisión Nacional del Litio, que declaró al mineral como "recurso estratégico". Esta declaración limitó la posibilidad de concesiones para la explotación y, al mismo tiempo, estimuló la formación de iniciativas público-privadas. Las empresas asentadas dirigen la explotación hacia su exportación para la comercialización de baterías, antes que otro tipo de proyectos que aporten valor agregado (Fornillo, 2019).

En el otro vértice del triángulo está Argentina, tercer exportador mundial del litio. Sus salares se localizan en las provincias de Jujuy, Salta y Catamarca. Cada una de ellas tiene una mirada particular respecto de la explotación del recurso (Quinteros, 2020). No obstante, casi todos los salares en Argentina se encuentran bajo manos de empresas privadas y transnacionales, con alguna participación de las provincias, como es el caso de Jujuy. ${ }^{5}$ Al no existir aún un marco legal nacional que regule la extracción de la salmuera de litio, esta se extrae sin rentabilidad para el Estado, más que la ganancia derivada de los impuestos que pagan las compañías por desarrollar sus actividades en el país (Juste, 2017b).

En el tercer vértice se encuentra Bolivia, en donde la presión de Potosí -departamento que se encuentra el salar de Uyuni- propició un proyecto que comprende desde la extracción del carbonato del litio hasta la concreción de la batería. La estrategia

\footnotetext{
${ }^{5}$ En Jujuy, para la aprobación de cualquier proyecto minero, la firma debe asociarse con JEMSE (Jujuy Empresa Minera Sociedad del Estado), la empresa estatal minera. Así, tanto la firma extranjera como la empresa local participan de la inversión y las ganancias (Quinteros, 2020).
} 
boliviana es exportar primero el potasio del salar a través de una empresa china y, en una segunda etapa, contar con recursos frescos y encarar la extracción de carbonato de litio. La gestión boliviana del litio aparece como la más completa, en el sentido de que incluye una cadena de valor en asociación con empresas chinas, una firma francesa que elabora materiales catódicos (elementos químicos procesados que conforman la batería) y una planta de ensamblado de baterías.

Las diferentes visiones acerca de la gestión de recursos naturales, como el litio, requiere ser considerada, además, desde la mirada de las unidades subestatales que poseen el mineral. Al analizar las perspectivas de estudio de la paradiplomacia, Oddone (2016) abordó la fragmentación de la unicidad de la política exterior, y con ella los modelos burocráticos y organizacionales que visibilizaron la democratización de los procesos de toma de decisión. Al focalizarnos en la gestión de los recursos naturales es inevitable tener en cuenta la división de facultades entre las unidades subestatales y el Estado para comprender la visión de las primeras.

Argentina, por ejemplo, es un Estado federal que reconoció a las provincias el dominio originario de los recursos naturales existentes en su territorio en el artículo 124 de la Constitución Nacional. En Bolivia, que adoptó la forma unitaria, la Constitución del Estado Plurinacional de 2009 reconoce la preexistencia de las naciones indígenas y su derecho a la participación en los beneficios de la explotación de los recursos naturales de sus territorios (Artículo 30, II. 16). A pesar de ello, las competencias del Estado Plurinacional son amplias y abarcan la gestión de los recursos naturales de carácter estratégico, como lo son los minerales. Los departamentos bolivianos tienen la facultad de promocionar las inversiones privadas en el marco de las políticas nacionales y la administración de sus recursos por regalías. Chile, por otro lado, es un Estado esencialmente unitario, en donde las regiones como Antofagasta y Atacama se deben ceñir a la política minera nacional. Por otra parte, como se mencionó antes, el Estado ha limitado las concesiones para la explotación desde 2015. Todo lo anterior obliga a reflexionar sobre la complejidad que implica el hecho de que en el triángulo del litio confluyan tres modelos y políticas diferentes para la gestión de la explotación del mineral.

Como se observa, el camino desde la extracción de la salmuera de litio hasta la producción de una batería es largo y complejo, cuestión que la región no puede afrontar en soledad. El verdadero valor parece hallarse en el conocimiento científico y en los medios que posibiliten alcanzar la producción de baterías, para lo cual el litio es un insumo básico, pero no el único. Fornillo (2019) plantea la necesidad de trabajar en el diseño de una "geopolítica del litio" que articule entramados industriales y genere políticas que coordinen a los países del triángulo del litio. Esto está aún lejos de realizarse, pero la coyuntura internacional podría propiciar el escenario esencial para un trabajo conjunto. Lograr una cadena de valor regional alrededor del litio permitiría romper con el patrón centro-periférico que ha caracterizado la relaciones entre Sudamérica y China, retrabajando la salmuera y evitando la exportación del carbonato de litio. De esta forma, confluyen en la ZICOSUR tres territorios con un gran potencial 
originado de la riqueza ambiental propia de la zona, donde la riqueza mineral plantea desafíos de inserción a un mercado cada vez con mayor demanda y especialización.

\section{El interés de China en la región}

Durante las últimas décadas, el sistema internacional ha presenciado un importante aumento de la demanda energética a nivel global. Esta tendencia se hizo especialmente notable en Asia, en respuesta al proceso de industrialización que impulsa el elevado crecimiento económico regional. Este proceso tiene como principal actor a China, cuya expansión económica es fácilmente visible, aunque no puede ser ignorado el rol de otros actores como India, Japón o Corea del Sur (Rubiolo y Baroni, 2019).

Autores como Rubiolo y Baroni (2019) coinciden en señalar que luego de la crisis del 2008, los países latinoamericanos ampliaron sus socios comerciales a fin de aumentar su autonomía, mejorar las condiciones de su inserción internacional y suavizar los efectos de la crisis. Entonces, la posibilidad de contar con más socios comerciales se presentó como la mejor opción para disminuir la vulnerabilidad de los cambios del contexto internacional.

La conjugación de condiciones domésticas de un inestable contexto económico internacional y de una creciente competencia entre países en desarrollo por cuotas de mercado e inversiones, favoreció la aproximación de América del Sur hacia China y, en menor medida, hacia las principales economías del Sudeste asiático. (Rubiolo y Baroni, 2019, p 13-14).

En 2010, China consiguió desplazar a los Estados Unidos como principal socio comercial de Sudamérica y pasó a ser el mayor inversor en la región. En 2013, el presidente Xi Jinping enunció la iniciativa "la Franja y la Ruta" (BRI, por sus siglas en inglés de Belt and Road Initiative), como proyecto de conectividad euroasiática. Esta propuesta comprende la Franja Económica de la Ruta de la Seda y la Ruta Marítima de la Seda del siglo XXI. Ambas vías conectan puntos estratégicos, según Oviedo (2018):

La Franja, como vía de comunicación terrestre, une a China con Europa a través de Asia central y occidental; y también conecta a China con el sudeste asiático, el sur de Asia y el océano Índico. La Ruta, como vía marítima, p arte de los puertos de seis provincias costeras de China (Jiangsu, Zhejiang, Fujian, Guangdong, Hainan y Shandong) en dos direcciones: 1) hacia Europa a través del océano Indico, el mar Rojo hasta llegar al Mediterráneo; y 2) hacia el Pacífico sur a través del Mar de la China Meridional y el Mar de la China Oriental. (p. 115).

La iniciativa presentó avances considerables en poco tiempo. En 2015, BRI expandió su ámbito geográfico hasta Oceanía, y en 2017 sumó a América Latina y el Caribe (ALC) cuando el presidente Xi consideró a la región como "extensión natural de la Ruta Marítima de la Seda del siglo XXI" (Oviedo, 2018, pp.124-125). De esta forma, la iniciativa adquirió características globales. 
El interés de los países asiáticos y, en particular de China en Sudamérica, es buscar recursos naturales para el crecimiento interno sostenible, así como el apoyo externo en un contexto de puja por el poder con Estados Unidos (Hongbo, 2014). De manera complementaria, China transita un proceso de diversificación de su matriz energética a través de la generación eléctrica por medio de las energías limpias, como son la solar, eólica, hidroeléctrica, celulosa y nuclear. Esta transición energética es un proceso de cambio estructural de fuentes de energía, en donde el vínculo con Sudamérica resulta estratégico, dadas las riquezas ambientales de la región.

En líneas generales, los intereses comunes consisten en la alta complementariedad en la esfera económico-comercial y energética. China, a medida que profundiza su inserción en la economía mundial, precisa importar gran cantidad de materias primas y productos para sostener su desarrollo económico. Por estos motivos, los países sudamericanos han cobrado relevancia como una fuente importante de recursos para Asia, pensando en sus minerales, recursos energéticos y productos agrícolas, entre otros.

En el caso de India, el interés en Sudamérica es más reciente. Su crecimiento ha estado, sin embargo, bajo la sombra del crecimiento chino. A pesar de ello, la coyuntura creó escenarios para un mayor acercamiento con Sudamérica. Los BRICS (Brasil, Rusia, India, China y Sudáfrica) y el Foro de Diálogo India-Brasil-Sudáfrica (IBSA) son muestras de ello. Aunque la presencia india es aún muy tenue en comparación con la de China, proyecta un futuro interesante.

La diferencia entre China e India en cuanto a su posicionamiento actual en Sudamérica reside en el comercio. Durante los últimos años, el desarrollo chino impulsó el comercio con nuestra región, transformándose en el principal comprador de los recursos naturales exportados. Como sostienen Bartesaghi y Bhojwani (2019), el vínculo empezó a través del comercio y luego se profundizó con una serie de acuerdos comerciales, de inversión, financiación y cooperación. Es de esperarse, entonces, que a medida que India continúe desarrollándose y posicionándose, empiece a competir con China en la región. Así como la presencia china en Sudamérica es parte de su modelo de desarrollo, India seguirá el mismo camino en los próximos años.

Desde la perspectiva de los países sudamericanos, la presencia de los países asiáticos a través de la demanda comercial y las inversiones se ha convertido en un factor importante para el crecimiento durante los últimos años. Los países de la región observan a los mercados asiáticos como potenciales posibilidades para acrecentar su exportación y al mismo tiempo para proveerse de la inversión externa y la cooperación tecnológica que posibiliten concretar obras de infraestructura y conectividad de desarrollo. La Comisión Económica para América Latina (CEPAL) misma ha manifestado que la economía china es el principal motor de crecimiento económico mundial y un mercado potencial para Latinoamérica (CEPAL, 2008).

Al respecto de esta complementariedad regional, Xiaoping (2014) sostiene:

China considera a América Latina como un socio estratégico en los asuntos de importancia global, en la reforma del sistema internacional actual y en el 
desarrollo económico mundial. Por su parte, América Latina ve a China como una importante fuerza emergente, de mucha influencia en el sistema internacional. (p. 31).

China trabaja en acelerar la estrategia de conversión económica y comercial, impulsando la formación de nuevos polos de crecimiento y elevando el nivel tecnológico sectorial y la eficiencia económica, junto con reforzar la competitividad internacional (Xiaoping, 2014). Asimismo, la región asiática se ha convertido en el principal origen de las inversiones en los territorios sudamericanos, cuestión no menos importante para pensar en relación con los procesos de modernización.

El crecimiento constante del gigante asiático, sumado al aumento de la inversión asiática en la región, ofrecen nuevas oportunidades para el desarrollo de las relaciones económico-comerciales entre ambas regiones. Según Xiaoping (2014), la clave del reequilibrio del intercambio económico-comercial entre ambas regiones reside en que, manteniendo los niveles de exportaciones latinoamericanos, China (e India) realicen más inversiones en la región, a través de transferencia de know-how, para mejorar la producción manufacturera y así cambiar los términos de intercambio con la región.

Para Argentina, Bolivia, Brasil, Chile, Paraguay, Perú y Uruguay, pero, por sobre todo, para las unidades subestatales de estos países que integran la ZICOSUR, esta complementariedad cobra un interés especial. Esto se debe a que las unidades subestatales zicosureñas son, en su mayoría, unidades de doble periferia, es decir, unidades subestatales que se sitúan en la periferia de un Estado que, al mismo tiempo, se encuentra en la periferia del sistema internacional (Juste, 2017a). En este sentido, las unidades subestatales de la ZICOSUR son economías relegadas, principalmente primarias y con escasa diversificación productiva, que se encuentran alejadas geográficamente de los principales centros de distribución y consumo estatal (Juste, 2017a). Por esos motivos, la complementariedad de intereses con Asia representa una oportunidad de diversificación productiva y asociación estratégica para obtener financiamiento para los proyectos de interés subestatal que no necesariamente pueden concretarse a través de los Estados nacionales.

En definitiva, China y sus pares de la región han sido claves en el incremento de las exportaciones primarias sudamericanas. Aunque, cabe destacar, las exportaciones sudamericanas consisten en productos primarios y sus derivados (Rubiolo y Baroni, 2019). Asimismo, esta primarización de las economías sudamericanas y su competencia con China en áreas industriales (especialmente con el MERCOSUR) plantean tensiones futuras de continuar esta tendencia.

\section{Áreas de asociación estratégica y principales obstáculos en la relación ZICOSUR-China}

Pensar en una vinculación estratégica entre la ZICOSUR y Asia obliga a repensar aquellos puntos o área de cooperación y conflicto que puedan existir, en 
relación con los intereses de cada parte. En especial, los intereses de la pujante China, actor que ha incrementado notablemente su presencia en la región mediante diferentes vías que analizaremos a continuación. Al respecto, Oviedo (2011) sostiene que:

La cooperación o el conflicto entre ZICOSUR y China emergerán de acuerdo al modelo de crecimiento que adopte el Estado al que pertenece la unidad subnacional, aunque coexistirán en el corto y mediano plazo, debido a la industrialización incipiente de los países latinoamericanos. (p. 18).

En el contexto de una aparente complementariedad de intereses entre ambas regiones, la tensión puede emerger a medida que se profundice el proceso de industrialización y se trabajen las cadenas de valor en la ZICOSUR. En este sentido, la modernización sudamericana puede propender a una relación más conflictiva con China, que necesita de vinculaciones con regiones que apuesten a la primarización de su comercio exterior (Oviedo, 2011).

Para las economías subestatales periféricas de un Estado en desarrollo, como las de las unidades subestatales que componen la ZICOSUR, la inversión extranjera representa un instrumento diferenciador y potenciador que les permite emprender proyectos productivos mientras asimilan tecnologías. Para modernizarse, las unidades subestatales de la ZICOSUR potencian la disponibilidad de sus recursos naturales para impulsar la obtención de inversiones y tecnología, sin dependerse necesariamente de los intereses de las unidades centrales de cada país (Buenos Aires, Santiago, La Paz, Brasilia, etc,) que limiten su desarrollo. Pero no todos los recursos naturales son inagotables y, por consiguiente, su utilización debe ser planificada y empleada conscientemente.

En este marco, las inversiones chinas en la subregión aparecen como el área de cooperación. La ZICOSUR dio la bienvenida a inversores chinos, incluso en el ámbito de los recursos no renovables. La mayoría de las inversiones están en este sector y generan un lobby chino cada vez más influyente en las decisiones de política nacional y subestatal (Juste, 2017b). El interés de empresas chinas se ha visto complementado no solo por el contexto internacional y regional, sino también por las políticas de promoción impulsadas desde la ZICOSUR y sus miembros, que bregaban por dar mayores facilidades a las inversiones. En Argentina, por ejemplo, el gobierno de la provincia de Salta propuso un programa de acompañamiento a las empresas que buscan invertir en litio; mientras que, en la provincia de Jujuy, el gobierno local ofreció beneficios impositivos en los primeros años y creó una empresa estatal para la conformación de sociedades público-privadas para la actividad minera.

En el caso de la producción del litio, se requieren inyecciones importantes de capital para que un proyecto obtenga niveles considerables de producción rentable y se expandan los beneficios al desarrollo local. El litio se visualiza como una nueva oportunidad de inserción de la ZICOSUR en el mundo en materia de minería, sector que ha posibilitado durante los últimos años la multiplicación de las señales de inversión para el desarrollo de proyectos en el norte de Chile y Argentina y en el sur de Bolivia. 
Como se precisó con anterioridad, el interés chino se enfoca en sus recursos naturales. Además del litio, otro tema que tracciona las inversiones chinas hacia Sudamérica tiene que ver con la producción de energías, especialmente, aquellas obtenidas mediante fuentes renovables. Por eso, distintas firmas e inversores apuestan a proyectos de energía solar, eólica, biomasa e hidráulica, inversiones que complementan las ligadas al litio.

China entiende que, para que Sudamérica y la ZICOSUR puedan ser socios capaces de responder a su creciente demandas, precisan mejorar sus condiciones energéticas y de conectividad. Sobre esta última, la conectividad, China ha desplegado inversiones relativas a la concreción de obras de infraestructura ferroviaria y la creación de carreteras viales que faciliten el transporte de la materia prima hacia los puertos y abaraten costos. Sin embargo, la infraestructura de conectividad es un tema amplio que no se pretende abordar en este trabajo.

Teniendo en cuenta la riqueza ambiental de la ZICOSUR, encontramos presencia de inversiones chinas en energías renovables y el litio que pasamos a examinar.

\section{Energías renovables}

Las energías renovables son aquellas que se obtienen de fuentes naturales inagotables. Se denominan así ya sea por la inmensa cantidad de energía que contienen, como por que son capaces de regenerarse por medios naturales. Son conocidas como energías limpias o alternativas y se obtienen de fuentes naturales como el sol, el agua, el viento y los residuos orgánicos. Constituyen son una alternativa viable a las energías convencionales, y su impacto ambiental es reducido.

En este sentido, la energía solar o fotovoltaica ha sido la que mayor inversión asiática ha recibido durante los últimos años, principalmente procedente de China. En 2016, la provincia de Jujuy (Argentina) presentó el proyecto "Cauchari", de producción de 300 megavatios (MW) de energía solar. Para su ejecución se asoció a la empresa Power China bajo la modalidad "Turn Key" o "llave en mano". La firma china estuvo a cargo del desarrollo de la ingeniería, la adquisición de la totalidad del equipamiento y suministros, y la construcción y puesta en marcha de las instalaciones.

El costo total del proyecto se estimó en U\$D 541 millones, de los cuales 357 millones correspondieron a la construcción de la planta fotovoltaica (Fenés, 15 de noviembre de 2017). Para financiar la puesta en marcha de Cauchari, Argentina y China firmaron un contrato gobierno a gobierno, con una tasa preferencial de 3\%, por el que Jujuy accedió a un crédito de U\$D 331 millones del Export-Import Bank de China, destinado a la construcción de la planta de energía fotovoltaica. Asimismo, para iniciar las obras y ofrecer certidumbre a sus socios extranjeros, Jujuy emitió un "bono verde" por USD 210 millones a una tasa del 8,625 \% anual (Juste, 2019). Cauchari, que inició su producción en agosto de 2020 en plena pandemia, se convirtió en el parque solar más 
grande de América, ubicado en plena puna jujeña, ${ }^{6}$ por encima de los cuatro mil metros de altura sobre el nivel del mar.

El norte chileno también presenta un gran potencial de producción de energía solar en el que los actores chinos se han mostrados interesados. Con más de $105.000 \mathrm{~km}^{2}$ de superficie, el desierto de Atacama cubre cinco regiones geográficas al norte de Chile. Esta zona se caracteriza por ser la más árida y con mayor radiación del mundo, y numerosos emprendimientos fotovoltaicos se ubican allí. Más del $70 \%$ de nuevos proyectos de energías limpias corresponden a solares fotovoltaicos. Este gran interés por los cielos y suelos chilenos lo han llevado a ganarse el apodo de "la Arabia Saudita de Latinoamérica". La principal razón para convertirse en un atractivo en los últimos años han sido los Pequeños Medios de Generación Distribuida (PMGD), consistentes en pequeños parques solares con una potencia instalada menor o igual a 9 MW (Juste, 2019).

Estas características se combinan con los bajos precios de los paneles solares provenientes de China. Las principales compañías chinas que actúan en el terreno son State Power Investment Corporation (por medio de Pacific Hydro), Southern Power Grid International y China Three Gorges (Vergara, 21 de agosto de 2019). A esto se suma que más de una decena de empresas ya han demostrado su interés por abrirse paso en el mercado local, especialmente apuntando a las licitaciones de suministro y mediante alianzas con actores domésticos.

Además, el departamento peruano de Moquegua, integrante de la ZICOSUR, ya cuenta con uno de los parques fotovoltaicos más grandes del mundo. La empresa Enel Green Power Perú (EGPP) invirtió cerca de U\$D 170 millones en la construcción del parque solar "Rubí". El proyecto está financiado en parte por recursos propios del Grupo Enel y por otra parte por el Banco Europeo de Inversiones (Singh, 13 de abril de 2018).

\section{Litio}

Según la CEPAL, Sudamérica registra desde 2006 un crecimiento paulatino como receptora de IED destinada en un 50\% al aprovechamiento de los recursos naturales. No obstante, los flujos hacia los recursos naturales en Sudamérica aumentaron considerablemente, debido al desmantelamiento de proyectos mineros en países desarrollados por el agotamiento de los yacimientos explotados, el endurecimiento de los requerimientos medioambientales, el alto nivel del precio de los commodities y las expectativas crecientes de las demandas de los países del BRIC (Juste, 2017b). La modernización económica de China, en especial, y los cambios en el orden internacional revistieron gran importancia para los Estados periféricos, tal cual señala Oviedo (2015),

\footnotetext{
${ }^{6}$ La puna es un área del altiplano o meseta de montaña que forma parte de la cadena montañosa de la cordillera de los Andes, caracterizada por ser una zona desértica y de altura. Se emplaza en el norte argentino y chileno y sur boliviano; en este caso, Cauchari se ubica en la puna jujeña.
} 
no solo produciendo vínculos sino también profundizándolos a través de su integración a la producción asiática.

Existen múltiples motivos por los cuales los inversores chinos han puesto la mirada en las reservas del triángulo del litio. Las unidades subestatales de la ZICOSUR, donde se encuentran los salares, poseen distinta legislación local (y nacional en función de su pertenencia a diferentes Estados), que hacen a algunas más interesantes que otras. Por un lado, existen razones de índole externo que tienen que ver con que la explotación del litio a privados no está autorizada en Bolivia, dada la política nacionalista de los recursos naturales impulsada por la gestión del presidente Morales; Chile, por su parte, no entrega más concesiones de litio a empresas privadas desde 1979. Así, Argentina quedó como el país más atrayente a las inversiones en el denominado "triángulo del litio" de la región.

Sin embargo, las condiciones para la explotación de carbonato de litio no son homogéneas en la extensión territorial del triángulo del litio. Las circunstancias restrictivas para la explotación del mineral en Bolivia y Chile propiciaron que las miradas de los inversores se voltearan hacia Argentina, que presentaba mayores ventajas comparativas y un marco jurídico que permitía explorar aquellos territorios que no lo habían sido con anterioridad. Con la llegada de Luis Arce a la presidencia de Bolivia se abrieron muchas interrogantes acerca del futuro del litio, esperándose que el Estado Plurinacional ponga en agenda la producción de salmuera y reestablezca una política de producción y valor agregado en torno a socios estratégicos (dirigida por el gobierno plurinacional) que en su momento inició Evo Morales.

Argentina continúa siendo, hoy por hoy, el país que presenta mayores ventajas. Esto es así porque en la división de facultades entre el Estado federal y las provincias, son estas últimas las que tienen a su cargo la gestión de sus propios recursos naturales. Y, en particular, las provincias del Noroeste argentino (Jujuy, Salta y Catamarca), que son las poseedoras del mineral, se han mostrado proclives a fomentar la llegada de inversiones que dinamicen sus matrices productivas, bajo diferentes modalidades de gestión. Estas provincias del norte argentino, que integran la ZICOSUR, lideran los proyectos de extracción de litio en asociación con capitales asiáticos

En Jujuy existen principalmente dos proyectos propiciados por inversiones, australianas y coreanas, que se asociaron a la empresa pública provincial Energía y Minería Sociedad del Estado (JEMSE):

a) El proyecto Olaroz-Toyota Tsusho: ubicado en el Departamento de Susques en la zona de puna o desierto, a 4.540 metros sobre el nivel del mar. El proyecto, iniciado en 2009, demandó una inversión de U\$D 330 millones y fue gestionado por un grupo inversor que conformó un joint venture del cual participaron: (i) La automotriz japonesa Toyota, a través de su empresa abastecedora Toyota Tsusho Corp, con 25\% del capital; (ii) la empresa australiana Orocobre, que opera en Jujuy a través de la empresa Sales de Jujuy S.A., que participó con 66,5\%; y (iii) la empresa minera estatal de Jujuy, JEMSE, participó con $8,5 \%$. La IED proveniente 
de la empresa Toyota por medio de Toyota Tsusho Corp alcanzó los U\$D 82,5 millones, siendo la empresa australiana Orocobre la mayor inversora, con casi U\$D 220 millones. A inicios de 2020, la empresa confirmó la firma de dos contratos con empresas chinas para la exportación de más de 10.000 toneladas por tres años, destinados a la producción de baterías para la industria tecnológica y automotriz (Juste, 2019).

b) El proyecto Olaroz-Cauchari-Lithium Americas: la inversión se originó luego de un convenio de cooperación firmado entre la empresa Lithium Americas Corp. y su empresa subsidiaria EXAR más la empresa coreana Posco. El documento marcó el inicio de la construcción de una planta piloto en 2014, con una inversión inicial de U\$D 425 millones (Juste, 2017b). La apuesta por el litio se reflejó en el fuerte financiamiento de las empresas propietarias. Posteriormente, se invirtieron U\$D 160 millones adicionales para la ampliación de la planta. Esta fue posible por la inversión de Ganfeng Lithium, que adquirió el $12,5 \%$ adicional de Exar, con lo cual esa compañía china tiene en la actualidad el $50 \%$ del proyecto y la otra mitad, la canadiense Lithium Americas.

En 2019, la provincia de Salta encabezó el ranking de anuncios de inversiones para la explotación de litio en la región, con U\$D 1.525 millones, provenientes de tres empresas. La firma china Ganfeng Lithium International proyectó U\$D 675 millones para el salar de Llullaillaco; la surcoreana Pohang Iron and Steel Company anunció U\$D 450 millones de inversión para producir hidróxido y carbonato de litio de material extraído en el salar del Hombre Muerto, y la firma francesa Eramet confirmó la inversión de U\$D 400 millones para los salares Centenario y Ratones.

En particular, la firma Posco es uno de los inversores más grandes proveniente de Corea del Sur, dedicado a la siderurgia, construcción, ingeniería y minería. Su más reciente objetivo es acelerar la producción de litio a escala comercial en Argentina, para de esa forma poder capitalizar la demanda mundial de materiales para baterías de vehículos eléctricos (Bellato, 11 de enero de 2019). Al margen de estos números impresionantes, la provincia concentra un centenar de proyectos exploratorios que continúan a la búsqueda de inversores.

Por su parte, el salar del Hombre Muerto, ubicado en límite entre las provincias de Catamarca y Salta, posee proyectos de extracción del litio, radicados en el salar para ambas provincias. En la provincia de Catamarca, la minera australiana Galan Lithium adquirió en 2020 el 100\% de las concesiones de las áreas que estaban siendo explotadas por la firma canadiense Portofino Resources.

Este es el único caso del norte argentino en el que la producción de carbonato de litio ha quedado fuera de los intereses asiáticos. También, a inicios de 2020, la provincia entró en contacto con el magnate Bill Gates, quien se mostró interesado en realizar un proyecto de extracción más "sustentable", en el salar de Antofagasta de la Sierra. El 
fondo Breakthough Energy Ventures, que dirige Gates, proyecta invertir unos U\$D 20 millones en un startup llamada Lilac Solutions, interesada en el proyecto de salmuera de litio Kachi, ubicado cerca de El Peñón, en el departamento de Antofagasta (Rodríguez, 1 de marzo de 2020).

Al margen de las inversiones antes analizadas, también se observan que otros proyectos de extracción de la salmuera de litio en estas provincias y en las regiones de Atacama y Antofagasta en Chile, que trabajan bajo inversiones canadienses y australianas, tienen como principal mercado de destino a China. Ejemplo de ello son los proyectos de Olaroz, Pozuelos, Maricunga de las firmas Orocobre, Toyota Tsusho y Jemse; LSC Lithium y Lithea; Lithium Power, MSB y Bearing Lithium, respectivamente.

\section{Conclusiones}

La importancia de la ZICOSUR como subregión económica radica en el potencial que le aportan sus recursos naturales. Son justamente estos los que le pueden posibilitar una inserción internacional que rompa el patrón centro-periferia que ha caracterizado históricamente las relaciones entre los países de Sudamérica y las potencias imperantes. De la gestión de estos recursos dependerá la forma y los términos de su inserción internacional.

Es innegable la atracción que la subregión despierta en China y, prueba de ello, son los proyectos de distinta índole que se han emprendido recientemente: iniciativas de desarrollo de energías renovables, infraestructura de conectividad multimodal y explotación del litio. El interés chino en la subregión parte de la necesidad de obtención de materias primas que son esenciales para mantener el crecimiento del otro lado del mundo. Tanto la región asiática, como la subregión de la ZICOSUR, enfrentan un proceso de modernización y consecuente crecimiento, aunque bajo modelos políticos y económicos diferentes.

En primer lugar, y a simple vista, podría pensarse en la inversión asiática como una vía de mantener la relación centro-periferia a través de las exportaciones de bienes primarios de la ZICOSUR (como la salmuera de litio), pero se observan otros elementos. Esta relación Norte-Sur muestra elementos de "complementariedad": a la exportación de materias primas y la importación de bienes industriales chinos, se suman importantes capitales asiáticos para proyectos de infraestructura de conectividad y acuerdos de cooperación. Estos elementos propiciaron que las unidades subestatales diseñaran estrategias locales que buscan maximizar los beneficios de las inversiones a través de la coordinación entre el Estado y el sector privado.

En segundo lugar, las inversiones chinas en la subregión continúan siendo fundamentales para el fortalecimiento interno del bloque. La inversión extranjera representa la posibilidad de generar proyectos energéticos, pero también proyectos de infraestructura y conectividad (ferrocarriles, carreteras, puertos, entre otros) que permitan mejorar los flujos de personas, bienes y servicios dentro de la ZICOSUR y, 
así, reducir costos de producción. Todo esto representa, para las unidades subestatales, una opción de financiamiento complementaria a la que pueden recibir de los gobiernos centrales, aporte fundamental para su desarrollo.

En tercer lugar, claramente el litio aparece como una nueva oportunidad para evitar el triste y conocido papel subregional de abastecer de materias primas a los países centrales y comprar productos terminados; esto es, lo que algunos autores llaman "caer en una neodependencia". Por eso, la importancia de integrar una cadena regional que aporte valor agregado y evitar así la exportación directa de la salmuera de litio. Es muy importante complementar la inversión y el know how extranjero con el sector privado y la universidad en la articulación de propuestas que desarrollen cadenas de valor. En este punto, el mayor obstáculo y desafío es coordinar una misma política en torno al litio, teniendo en cuenta las asimetrías existentes entre las unidades subestatales en donde el recurso se encuentra y la mirada dispar de los Estados centrales. La clave estaría en confluir los intereses estatales y subestatales alrededor de una misma política subregional y regional en torno al litio.

En cuarto lugar, y vinculado con el punto anterior, la aparente complementariedad y sinergia entre China y la ZICOSUR puede verse alterada si el bloque zicosureño consigue alcanzar una política que articule una cadena de valor del litio a largo plazo. Esto rompería el patrón de centro-periferia que caracteriza la relación, y obligaría a China a replantearse los términos y condiciones de la vinculación.

En quinto y último lugar, la incógnita restante es el rol futuro de la pujante India en Sudamérica. De crecer su presencia en la región, competiría con China por recursos y mercados. Esto podría desembocar en una competencia entre las unidades subestatales de la ZICOSUR por captar mercados e inversiones. Una primera consecuencia de ello sería el debilitamiento del bloque subregional. Las opciones posibles parecen ser escenarios de tensión o cooperación entre las unidades subestatales, que dependerán de la capacidad de la ZICOSUR para diseñar estrategias unificadas frente a terceros que fortalezcan la subregión y no la desintegren.

Retomando las preguntas que guiaron este análisis, los recursos naturales de la ZICOSUR -junto a su necesidad de atraer inversiones que le permitan alcanzar sus objetivos de desarrollo- resultan complementarios a los intereses de China en Sudamérica, que busca expandir su área de influencia y asegurarse los medios que le permitan continuar su crecimiento. No obstante, el vínculo actual se caracteriza por ser una relación entre una subregión periférica, la ZICOSUR, y un actor central como China.

La explotación de las riquezas naturales de la ZICOSUR puede ser una oportunidad de desarrollo a largo plazo si logra articularse una cadena de valor regional a través de, por ejemplo, el litio. Los modelos actuales de producción son diferentes y necesitan de altos grados de inversión y desarrollo tecnológico que aporten valor agregado. El desafío está en consensuar un único modelo que articule capacidades y se oriente a industrializar la salmuera del litio, impulsado por la inversión china. 


\section{Referencias bibliográficas}

Álvarez, M. (2017). El rol de la paradiplomacia en las entidades binacionales: análisis del accionar de las provincias argentinas y regiones chilenas en los casos de EBITAN y EBIFETRA. Si Somos Americanos. Revista de Estudios Transfronterizos, 17(2), 77-95. Recuperado de https://www.sisomosamericanos. cl/index.php/sisomosamericanos/article/view/776

Bartesaghi, I. y Bhojwani, D. (2019). La negociación Mercosur-India: oportunidades comerciales para Uruguay. Estudio de Asia y África, 54(2), 329-360.

Bellato, R. (11 de enero de 2019). La minera Posco aumentó un \% sus reservas de litio en Salta. Ecojournal. [En línea]. Recuperado de https://econojournal. com.ar/2019/11/la-minera-posco-aumento-un-150-sus-reservas-de-litio-en-salta/

Calvento, M. (2014). La inserción internacional de los actores subnacionales: análisis de un proceso contemporáneo. Revista Interaçoes, 15(2), 301-313.

Centro Regional de promoción de la MIPYME (CENPROMYPE), Comisión Económica para América Latina y el Caribe (CEPAL) y Secretaría de Integración Económica Centroamericana (SIECA) (2018). Articulación productiva y cadenas regionales de valor: Una propuesta metodológica para la región SICA. Ciudad de México: CEPAL. Recuperado de: https://www.cepal.org/es/publicaciones/44070-articulacion-productiva-cadenasregionales-valor-propuesta-metodologica-la

Comisión Económica para América Latina y El Caribe (CEPAL) (2008). Las relaciones económicas y comerciales entre América Latina y Asia-Pacífico. El vínculo con China. Segunda Cumbre Empresarial China-América Latina (documento). Harbin, Heilongj, China, 20 y 21 de octubre. Recuperado de https://www.cepal.org/sites/default/files/publication/files/2912/LCL2959_es.pdf

Constitución del Estado Plurinacional de Bolivia [Const.]. Art. 30, II. 16. Febrero de 2009 (Bolivia).

Constitución Nacional de la República Argentina [Const.]. Art. 124. 23 de agosto de 1994 (Argentina).

Duchacek, I. (1990). Perforated sovereignities: Towards a typology of new actors in International Relations. En H. Michelmann y P. Soldatos (eds.), Federalism and International Relations: The Rol of Subnational Units (pp. 1-33). Oxford: Clarendon Press.

Fenés, G. (15 de noviembre de 2017). Los detalles sobre el Parque solar "Cauchari”: ¿se aplicará el take or pay? Energía Estratégica. [En línea]. Recuperado de http://www.energiaestrategica.com/costos-plazos-financiamiento-del-parquesolar-cauchari-se-aplicara-take-or-pay 
Fornillo, B. (coord.) (2019). Litio en Sudamérica. Geopolítica, energía y territorios. Buenos Aires: IEALC (Instituto de Estudios de América Latina y el Caribe), Editorial El Colectivo y CLACSO.

Gourevitch, P. (1996). La segunda imagen invertida: los orígenes internacionales de las políticas domésticas. En O. Gil y L. Sanz (comps.), Las fuentes internacionales de las políticas domésticas (pp. 21-68). Madrid: Zona Abierta.

Hongbo, S. (2014). Modelo de cooperación energética entre China y América Latina. Problemas de Desarrollo, 45(176), 9-30.

Juste, S. (2017a). La condición de doble periferia en unidades subestatales. Revista INTERAÇÕES, 18(4), 169-184.

Juste, S (2017b). La IED de países del noreste asiático en la producción minera de la provincia de Jujuy: el litio como recurso estratégico para el desarrollo local (2010-2016). En E. Oviedo, Inversiones de China, Corea y Japón en Argentina: análisis general y estudio de casos (pp. 261-275). Rosario: UNR Editora.

Juste, S. (2019). Las relaciones argentino-china desde la perspectiva de los actores subestatales. Intellector, 16(32), 28-40.

Juste, S. y Oddone, N. (2020). Aportes teóricos para el estudio de la cooperación transfronteriza de unidades subestatales de doble periferia. Cuadernos de Política Exterior Argentina. Nueva Época, 132, diciembre, 63-78.

Keohane, R. y Nye, J. (1989). Poder e interdependencia. La política mundial en transición. Buenos Aires: Grupo Editor Latinoamericano.

López, A., Obaya, M., Pascuini, P. y Ramos, A. (2019). Litio en la Argentina. Oportunidades y desafíos para el desarrollo de la cadena de valor. Buenos Aires: Secretaría de Ciencia, Tecnología e Innovación Productiva-Ministerio de Educación, Cultura, Ciencia y Tecnología de Argentina. Presidencia de la Nación-Banco Interamericano de Desarrollo. Recuperado de: https://www.argentina.gob.ar/sites/default/files/bid-litio-final.pdf

Maira, L. (2010). La política internacional subnacional en América Latina. Buenos Aires: Libros del Zorzal.

Manning, B. (1977). The Congress, the Executive and Intermestic Affairs: Three proposals. Foreign Affairs, 55(2), 306-324.

Oddone, N. (2016). La paradiplomacia desde cinco perspectivas: reflexiones teóricas para la construcción de una comunidad epistémica en América Latina. Revista de Relaciones Internacionales, 89(2), 47-82. Recuperado de https://doi.org/10.15359/ri.89-2.2

Oviedo, E. (2011). La proyección de la ZICOSUR en el mundo: el horizonte de cooperación y rivalidad económica con China. I Simposio Electrónico Internacional sobre Política China, 1-21 de marzo. Observatorio de la Política 
China, España. Recuperado de https://www.igadi.gal/china/2011/ pdf/edo_la_proyeccion_de_la_zicosur.pdf

Oviedo, E. (2015). El ascenso de China y sus efectos en la relación con Argentina. Revista Estudios Internacionales. Universidad de Chile, (180), 69-70.

Oviedo, E. (2018). Oportunidades, desafíos e intereses de Arxentina na OBOR. Tempo Exterior, 19(37), 115-129.

Padilla Pérez, R. y Oddone, N. (2016). Manual para el fortalecimiento de cadenas de valor. México: CEPAL y FIDA (Fondo Internacional para el Desarrollo Agrícola). Recuperado de: https://www.cepal.org/es/publicaciones/40662manual-fortalecimiento-cadenas-valor

Perkmann, M. (2003). Cross border regions in Europe. Signifcance and drivers of regional cross-border cooperation. European Urban and Regional Studies, 10(2), 153-171.

Quinteros, V. (2020). Cadena regional de valor en torno del litio: posibilidades dentro de los procesos de integración latinoamericanos. (Tesis de maestría en Procesos de Integración Regional, Facultad de Ciencias Económicas. Escuela de Estudios de Posgrado, Universidad de Buenos Aires, Buenos Aires, Argentina). Recuperado de: http://bibliotecadigital.econ.uba.ar/download/tpos/15021601_QuinterosVJ.pdf

Rhi-Sausi, J. L. y Oddone, N. (2009). Cooperación transfronteriza e integración en América Latina. En J. L. Rhi-Sausi y D. Conato (coords.), Fronteras $e$ integración transfronteriza en el Mercosur. (pp. 37-54). Roma: Centro Studi di PoliticaInternazionale e Istituto Italo-Latino Americano.

Rodríguez, G. (1 de marzo de 2020). Litio en Catamarca: entre el interés de Bill Gates y la lucha ambiental de las comunidades. Tiempo Argentino. [En línea]. Recuperado de https://www.tiempoar.com.ar/nota/litio-en-catamarca-entre-elinteres-de-bill-gates-y-la-lucha-ambiental-de-las-comunidades

Rubiolo, M. F. y Baroni, P. (2019). El este de Asia como socio de América Latina: implicancias económicas y sociales de una inserción asimétrica en los casos de Chile y Argentina. PORTES, Revista Mexicana de Estudios sobre la Cuenca del Pacífico. Tercera Época, 13(25), 7-32.

Singh, N. (13 de abril de 2018). Está operativo el Parque Solar Rubí, uno de los proyectos más grandes de Enel en Perú. Energía Estratégica. [En línea]. Recuperado de https://www.energiaestrategica.com/esta-operativo-el-parquesolar-rubi-el-proyecto-mas-grande-de-enel-en-peru/

Valle Sosa, M. V. (2012). La emergencia de los actores locales en el plano internacional y los procesos subnacionales de integración regional: El caso de la ZICOSUR. Revista TIP. Trabajos de Investigación en Paradiplomacia, (3), 75-86. 
Venegas San Martín, F. (2019). Pensar la integración regional desde los márgenes: la importancia y desafíos de la dimensión subestatal en América Latina desde la experiencia chileno-argentina. Si Somos Americanos. Revista de Estudios Transfronterizos, 19(2), 69-88. Recuperado de https://www.sisomosamericanos. cl/index.php/sisomosamericanos/article/view/924

Vergara, T. (21 de agosto de 2019). Inversiones chinas en sector energía superan los US\$ 2.500 millones. El Mercurio Inversiones. Recuperado de https://www.elmercurio.com/Inversiones/Noticias/Analisis/2019/08/21/Inversion es-chinas-en-sector-energia-superan-los-US-2500-millones.aspx

Xiaoping, S. (2014). Historia de las relaciones entre China y América Latina. En L. Bogado Bordazar (ed.), Las relaciones entre China y América Latina y los enigmas de los lazos históricos (pp. 17-56). Ciudad de la Plata: Editorial IRI.

Zona de Integración del Centro Oeste de Sudamérica (ZICOSUR) (23 de diciembre de 2020). Mapa de ZICOSUR. [En línea]. Recuperado de http://zicosur.co/zona-deintegracion/ 